\title{
Ring-fencing a budget for cancer drugs: is it fair?
}

\author{
${ }^{1} \mathrm{~J}$ Graham, ${ }^{2} \mathrm{~J}$ Cassidy, ${ }^{3} \mathrm{D}$ Hughes, ${ }^{4} \mathrm{M}$ Duerden \\ ${ }^{1}$ SpR Medical Oncology, Beatson West of Scotland Cancer Centre, Glasgow; ${ }^{2}$ CRUK Professor in Medical Oncology, Glasgow University, \\ Glasgow; ${ }^{3}$ Professor of Pharmacoeconomics, Centre for Health Economics and Medicines Evaluation, Institute of Medical and Social Care \\ Research, Bangor University, North Wales, UK; ${ }^{4}$ Assistant Medical Director, Betsi Cadwaladr University Health Board, Honorary Senior \\ Lecturer, Bangor University, North Wales, UK
}

Ring-fencing is defined as protecting funds for use in a specific area. In the National Health Service in the UK, various methods to ring-fence cancer have been employed over the years; more recently the Cancer Drugs Fund in England has enabled cancer drugs that would not normally be considered cost-effective to be provided to patients. This has created variation in provision between England and the devolved countries. While some would argue that ring-fencing allows major advances to be made in the treatment of a particular condition, others would argue that it is intrinsically unfair. In this debate, Graham and Cassidy have written an article arguing the affirmative position and Hughes and Duerden were invited to respond directly to their arguments. As with all the RCPE's 'Current controversies', the authors have been asked to take a deliberately polarised position and so the views they express may be somewhat overstated.

KEYWORDS Cancer, medicines, funding, NHS, equity, economics

DECLARATION OF INTERESTS Dr Graham, Professor Hughes and Dr Duerden have no conflicts of interest to declare. Professor Cassidy has taken up a post with Roche USA.

\begin{abstract}
Correspondence to
DA Hughes,

Professor of Pharmacoeconomics

Centre for Health Economics and

Medicines Evaluation,

Institute of Medical and Social

Care Research, Bangor University,

Dean Street, Bangor LL57 IUT, UK
\end{abstract}

tel. +44 (0) I 248382950

e-mail d.a.hughes@bangor.ac.uk

\section{J Graham, \\ Beatson West of Scotland \\ Cancer Centre \\ 1053 Great Western Road \\ Glasgow GI2 OYN, UK}

tel. +44 (0) I4I 30 I 7000

e-mai janet.graham@ggc.scot.nhs.uk

\section{Ring-fencing a budget for cancer drugs: is it fair? For}

\section{J Graham, J Cassidy}

We will argue that ring-fencing of funds for cancer is a sensible and correct approach. As a preamble (and to deflate the argument that all diseases should be treated equally), we need to be clear why cancer is different from other diseases. The diagnosis of cancer immediately raises the next question 'how long have I got?'. This is not the response one gets when telling a patient they have a myocardial infarction or diabetes - even though these diseases also carry a lethal threat. Why is this? In part, it stems from how we report the results of cancer trials $-x$ year survival, median time to death, etc. These statistics exist for other diseases but are rarely if ever discussed with patients. We believe that the current way we report results of cancer drug studies consistently undervalues the worth of these agents. Median survival means little or nothing to an individual. In addition, the median statistic hides the patients who do very well. So we regularly have the discussion 'is $\mathrm{x}$ weeks more survival worthwhile?'. A better way of discussing results with patients would be to say $x \%$ gain nothing, $y \%$ gain a little and $z \%$ gain a lot. Then patients could decide if the gamble to be part of $\mathrm{z}$ was a worthwhile thing to do.

This complicates the 'standard' methodology of describing worth by quality adjusted life years, so-called 'QALYs'. This measure is commonly used by funding bodies, such as the National Institute for Health and
Clinical Excellence (NICE), to make funding decisions and is defined by them as a measure of a person's length of life weighted by a valuation of their health related quality of life. However the whole concept of QALYs is fundamentally flawed in any case. As an example, if you lose a leg then your quality of life (QoL) will go down. But do all one-legged people then have a low QoL for the rest of their lives - no. They 'acclimatise' and regain some or all of the lost $\mathrm{QoL}$ with time.These calculations also take no account of the savings that can be derived from treating a patient - for example reduced hospital admissions, reduced requirements for analgesics, antiemetics etc.

Unfortunately there is nothing new about our underspending on cancer patients. Nearly 15 years ago Leonard and colleagues reported in the BMJ that Britain was spending 10 -fold less than the US and 3.5 -fold less than the rest of Europe on cancer care and sadly that situation has not improved. Despite our best efforts to treat our patients in an evidence-based manner and within a constrained National Health Service (NHS), our patients still do significantly worse than in other countries. In a recent study published in The Lancet, there was a more than $12 \%$ difference in survival rates between the lowest performer, the UK, and the highest performer, Australia, for patients with colorectal cancer. 
In the case of lung cancer, there was only an $8.8 \%$ fiveyear survival for UK patients compared with $18.4 \%$ in Canada.The differences in survival rates were particularly striking for patients of more than 65 years of age and this is clearly concerning, given our noticeably aging population.'

There is a common misconception that we would blow the whole NHS budget if we spent money on the appropriate treatments for our cancer patients but this is just not the case. Richards et al found that only $8 \%$ of the total cancer care budget for a given inpatient was on chemotherapy (compared to 13\% for laboratory and radiological investigations) and yet no one is suggesting we stop performing routine investigations on our patients. ${ }^{2}$ It was estimated a few years ago that while approximately $f 170 \mathrm{~m}$ a year was being spent on anticancer drugs, including a total chemotherapy drug bill of only $₫ 58 \mathrm{~m}$, that by comparison $£ 250 \mathrm{~m}$ was being spent on just one particular brand of proton pump inhibitor. ${ }^{3}$ And the really important thing to remember about all of this is that spending money does save lives. According to Department of Health figures, we spend approximately $£ 80$ on cancer per head of population (per year) and have an overall five-year survival for men of $36 \%$ and women of $47 \%$. In Germany, nearly twice as much is spent $(€ \mid 43)$ and this translates into an additional 10\% five-year survival ( $45 \%$ for men and $55 \%$ for women).

So ring-fencing is protective. We would therefore argue that it is good sense to protect the cancer budget. It should not be diverted or frittered away in less dangerous (no matter how common) diseases states. Not many other diseases require such intensive and complex management involving surgeons, pathologists, radiotherapists, etc all working in a team. The argument then becomes how big a budget do we need for cancer? In the UK we spend more money on anti-diarrhoeal drugs than cytotoxics. These are mainly prescribed for selflimiting episodes - so we would argue ring-fencing should also be used to stop money leaking away in such a wasteful way. The fence keeps stuff in and other stuff out.

Our colleagues on the other side of the argument will attempt to persuade you that cancer should not be treated in a different way to any other illness, that all the drugs are expensive, that many of them only give patients extra weeks or months... that ring-fencing simply takes money from one system and puts it into another - a system that is less rigorously tested than usual with the current drug funding decision makers: NICE and the Scottish Medicines Consortium (SMC). But the point is that the current system fails patients and therefore until we have a better one, we need a fall-back for our patients. We are there to serve the patient's best interests always. There are numerous examples where drugs with stunning results for patients - real breakthroughs in the battle against cancer - have been unjustly not approved by our regulatory bodies (discussed in more detail below). Of course injustice is a relative phenomenon. But I dare say if any of our readers were unlucky enough to contract the cancers in question they would wish to get the best drugs - in fact, we know that from treating medical colleagues.

Cancer is not only a disease of the elderly - increasingly we see young patients with solid tumours. The big difference between this and the haematological malignancies is that the prognosis is extremely poor. For many years we have made small steps improving survival using changes in the dosing, scheduling and combinations of traditional chemotherapies but the real breakthroughs have come from the novel targeted agents.

Response Evaluation Criteria in Solid Tumours (RECIST) computerised tomography (CT) is the common method of assessing response to treatment. Essentially, index lesions are chosen and the sum added up and then response is divided into four subgroups: complete response, partial response, stable disease and progressive disease. However, these agents are often cytostatic (stop the cancer growing) rather than tumour shrinking and many doctors and patients would argue that long-term stable disease is much more meaningful than a tumour shrinkage which is then short-lived.Therefore measuring responders as only those with tumour shrinkage may no longer be appropriate. Instead, physicians are increasingly using waterfall plots (which show the change in size of each individual tumour rather than splitting them into these arbitrary categories) to illustrate the effectiveness of novel agents and it is clear from examining these that although only a small percentage of patients may make the cut-off for a complete or partial response, that a huge number benefit from the treatment.

For other drugs it is their stunning effectiveness that is their downfall. In the case of sunitinib, it was not approved by NICE for renal cancer in late 2008, despite a more than doubling in Progression Free Survival (PFS) (II versus five months) and a six-month (26 versus 20 months) advantage in overall survival, ${ }^{4}$ because the authors failed to demonstrate a statistically significant overall survival advantage... but the reason the overall survival advantage was not possible to demonstrate was because the experimental drug was so effective that the study was unblinded and many of the patients who were not randomised to receive it as part of the study were allowed to cross over and have it. There is really no way around this - as clinicians it would be extremely hard to deny a patient an effective treatment simply because it was going to muddy our clinical trial endpoint.

There is no doubt that the system as it stands fails our patients. Major reorganisation is planned for the NHS, but it is highly doubtful that the proposed reorganisation is going to improve things in the short-term. In an ideal world 
adequate funding would be available so that we could assess our patients in a fair and non-postcode determined manner and provide the treatment that is needed - but until that day comes ring-fencing needs to stay.

\section{REFERENCES}

I Coleman MP, Forman D, Bryant $\mathrm{H}$ et al. Cancer survival in Australia, Canada, Denmark, Norway, Sweden and the UK, 19952007 (the International Cancer Benchmarking Partnership): an analysis of population-based cancer registry data. Lancet 201 I; 377: 127-38. doi: 10.1016/S0|40-6736(I0)62231-3

2 Richards MA, Braysher S, Gregory WM et al. Advanced breast cancer: use of resources and cost implications. Br J Cancer 1993; 67: 856-60. doi: 10.1038/bjc. 1993.157
3 Leonard RC, Smith IE, Coleman RE et al. More money is needed to care for patients with cancer. BMJ 1997; 315:8II-12.

4 Motzer RJ, Hutson TE, Tomczak $P$ et al. Sunitinib versus interferon alfa in metastatic renal-cell carcinoma. N Engl J Med 2007; 356: I 15-24. doi: I0.1056/NEJMoa065044

\section{Ring-fencing a budget for cancer drugs: is it fair? Against}

D Hughes, M Duerden

Decisions concerning the funding of treatments - cancer treatments in particular - evoke strong emotions. Negative recommendations by the National Institute for Health and Clinical Excellence (NICE) are viewed by some as being detrimental to patient care, denying patients with real clinical needs access to effective therapies. The UK coalition Government has seemingly sympathised with this view, although endorsing the overall role of NICE, by introducing the Cancer Drugs Fund for England in 2010.The purpose of the scheme was to ring-fence $£ 200 \mathrm{~m}$ per annum of existing National Health Service (NHS) funds to enable clinicians to prescribe treatments that have not been recommended by NICE.' However, concern about the plight of desperate patients, alongside the promise of targeted treatment, makes it easy to lose sight of the necessity to rigorously appraise experimental treatments. ${ }^{2}$ It is imperative, therefore, to ensure that both current and future patients, and also society, are provided with safe, effective and cost-effective interventions. Alas, the granting of marketing authorisation ('the license') does not give such a guarantee.

Decisions made by NICE are based on rigorous assessments of the clinical and economic evidence, and negative recommendations (made in around 13\% of all appraisals) are only issued if there is a lack of evidence for clinical effectiveness or if the medicine is not considered to be a cost-effective use of NHS resources, compared with current NHS practice. The Cancer Drugs Fund is therefore used to buy treatments that are not deemed by NICE to be clinically effective, or cost-effective and seems to undermine the role of NICE in its technology appraisal of cancer drugs.'

In our counterpoint, we provide a public health perspective to resource allocation, discuss the implications of the English Cancer Drugs Fund, and correct some common fallacies concerning health economics.
How effective are new medicines for cancer? Among the ten new molecular-targeted drugs approved by the Food and Drug Administration (FDA) between 2000 and 2010 for the treatment of metastatic adult solid tumours, none are curative though some may extend life, but by no more than a median of five months. ${ }^{3}$ It is important to recognise that if one of these drugs extends survival by a median of five months, $50 \%$ of people receiving that treatment get less than this advantage. With costs typically in the order of tens of thousands of pounds per patient (for example, lenalidomide costs around $€ 30,000$ - or about $£ 200$ per capsule), it is hardly surprising that most exceed the NICE cost-effectiveness threshold (representing the marginal value of health) of $£ 20,000$ to $£ 30,000$ per qualityadjusted life-year (QALY). To enable more drugs to be used, Patient Access Schemes were introduced to discount the costs of very expensive treatments to the NHS but even with these, some drugs exceeded the upper $£ 30,000$ per QALY threshold. Partly for this reason, an adjustment was made in 2009 to allow some cancer drugs to be provided at an even higher cost-effectiveness threshold using the NICE end of life criteria. ${ }^{4}$ This threshold appears in practice to be around $£ 50,000$ per QALY. However the Cancer Drugs Fund now allows use of drugs in the English NHS that exceed even this very high threshold.

The problem with the Cancer Drugs Fund and with applying end of life criteria is that they allow drug companies to charge what they think the market can bear and do little to exert downward pressure on drug pricing in the UK. In any case, drug companies are reluctant to set lower drug costs as the UK is often used as a 'reference price' to set prices in other countries. From 20I4, a valuebased pricing mechanism will be introduced (and with it the Cancer Drugs Fund will end), with the intent of enabling new medicines to be priced according to the health benefits generated. If companies decline to supply 
a medicine at a price that relates to its value to the NHS, it will be their responsibility - not NICE's - to explain why. This may change the current situation where NICE is seen as the bad guy and profit-maximising drug companies are seen as the innocent victims. The media and patient support groups accuse NICE of denying access to treatment but few criticise the extreme costs of treatment which may give little benefit to many who receive them.

Should cancer be a special case? Perhaps so, but fundamentally, this is an empirical question that needs to be asked of the general public. The activity of media and patient support groups may suggest that society expects this preference but this may not be the case. The counter view is that there are equally deserving patients with other diseases who may not have such vocal advocacy. Few would argue that patients with New York Heart Association (NYHA) functional class III/IV congestive heart failure are in better states of health, or have better prognoses than many patients with cancer. Also, there are obvious differences among the cancers, in terms of life expectancy, and the quality of life patients experience. It may be that short gains in survival for some of these cancers are at the expense of considerable additional suffering. In these instances the additional costs of treatment might be better invested in enhancing palliative care service.

If the answer is that, overall, cancer should be a special case, then a year of life gained in a person with cancer should clearly be valued higher than a year of life gained in a patient with some other illness. In general, NICE takes the view that the QALY, its preferred measure of health outcome, should be valued equally, irrespective of disease, age, gender or any other characteristic. However there are some exceptions. As already stated, concerning end of life criteria, NICE values QALYs gained with treatments that extend life expectancy in terminal diseases higher than other QALYs. In certain other instances, it can also place higher value on treatments of severe conditions, treatments that are innovative, and treatments for children or disadvantaged populations. ${ }^{6}$ The NHS consequently pays more for the marginal additional health benefits achieved by treatments such as lenalidomide (for multiple myeloma), sunitinib (for advanced renal cancer), pemetrexed (for malignant mesothelioma), imatinib (for chronic myeloid leukaemia), and trastuzumab (for advanced breast cancer). As NHS resources are finite, for each QALY gained, more than one QALY is lost through the displacement of other services necessary to fund these treatments - resulting in an overall reduction in population health gain.

In the case of cancer treatments not recommended by NICE, such as cetuximab for the treatment of squamous cell cancer of the head and neck, which was considered to be substantially less cost-effective than what is normally considered to be an acceptable use of NHS resources, ${ }^{7}$ funding from the Cancer Drugs Fund would represent an even greater reduction in population health. It seems to us wholly inappropriate and inequitable to use scarce resources to buy these inefficient treatments in the NHS in England at the expense of the health of the NHS population as a whole. Furthermore, in order to qualify for access to the Cancer Drugs Fund in England, these treatments need to have been turned down in the Strategic Health Authority area because an exceptional, individual case has not been made.

How do NICE recommendations compare internationally? Compared with Canada and Australia, where the clinical and cost-effectiveness of treatments is also assessed, NICE is generous. It recommended $87 \%$ of its appraised medicines, compared with $50 \%$ and $54 \%$, respectively. ${ }^{8}$ However, focusing only on cancer drugs, and compared with health insurance providers in the USA, positive coverage decisions were made by NICE in $39 \%$ of cases, compared with $100 \%$ in the USA. ${ }^{9}$ Of course, not all Americans are covered by health insurance (about 17\% are not), and all patients are subject to substantial co-payments - which can cost thousands of dollars each month. Following the recommendations of the Richards report on improving access to cancer medicines, ${ }^{10}$ patients may now pay privately for 'top up' treatments which are not available on the NHS if they desire this, and can afford it.

Is it unethical to ration resources in the NHS on the basis of cost? Our view is that with finite healthcare budgets, it is unethical not to pay attention to costs as well as overall need; we all have a duty to make the best use of public money and finite resources. A hypothetical example looking at overall population need is seen with vaccines. Suppose that $f \mathrm{Im}$ is available for a vaccination programme and two potential vaccines are available: vaccine $A$ is $95 \%$ effective, and costs $£ 5$ per dose, while vaccine $B$ is $99 \%$ effective but is twice the cost $(f \mid 0)$. Individuals might reasonably prefer B. However, from a payer's perspective, EIm would buy 200,000 doses of vaccine $A$ and offer protection to 190,000 patients. Given the budget available, only 100,000 doses of the more effective vaccine B may be purchased, to protect 99,000 individuals. Thus, when costs are considered, the seemingly most effective intervention for the individual is inferior from a population perspective as only about half the number of people are immunised. The same principle applies to all health technologies, procedures, services and interventions, cancer treatments included.

Is a health economic appraisal based on the QALYs fundamentally flawed? If it is, accepting the need for decisions on the availability of new medicines to be informed by health economics, the next challenge would be to define a common outcome measure for making valid comparisons across different treatments and diseases. Our view is that the QALY is well suited to the task. For example, a drug which improves lung function, and which 
increases the time without the symptoms of asthma cannot be compared directly - on an asthma symptomrating scale - with a drug for cancer which might extend life expectancy. The advantage of the QALY is that it is a measure which combines both quality and quantity of life into a single index and enables such comparisons. In constructing the QALY, times spent in states of health are valued on a scale of 0 to I ( 0 representing death; I representing full health), and summed over an appropriate time horizon. Symptomatic asthma, for instance, might be valued at 0.8 , compared with 0.95 during times without symptoms. A treatment which improves health by 0.15 for six months (on average) over a lifetime would result in a QALY gain of $0.075\left(=0.15^{*} 6 / 12\right)$. Compare this with a treatment for cancer which extends life by two months but, because of adverse events, reduces quality of life from 0.5 to 0.45 during the six months of treatment. The associated QALY gain is $0.058(=0.45 * 6 / 12-0.5 * 4 / 12)$. From a QALY perspective, the treatment for asthma is marginally more effective than the treatment for cancer. From an economic perspective, the pertinent issue is whether these gains in health are greater than the benefits forgone from the displacement of other NHS activities required to fund them. Economic evaluations consider the net cost of treatment and associated tests, procedures and management of adverse events, less the savings of reduced hospitalisation, GP appointments etc.

\section{REFERENCES}

I Duerden M. From a cancer drug fund to value based pricing of drugs. BMJ 2010; 341:c4388. doi: 10.11 136/bmj.c4388

2 Miller FG, Joffe S. Balancing access and evaluation in the approval on new cancer drugs.JAMA 20I I;305:2345-46. doi:10.100I/jama.20I I.784

3 Ocana A, Tannock IF. When are 'positive' clinical trials in oncology truly positive? J Natl Cancer Inst 20 I I; 103:16-20. doi:10.1093/jnci/ djq463

4 National Institute for Health and Clinical Excellence. Appraising life-extending, end of life treatments. London: NICE; 2009. [cited 201 I June 12]. Available from: http://www.nice.org.uk/media/88A/ F2/SupplementaryAdviceTACEoL.pdf

5 Hughes DA.Value-based pricing: incentive for innovation or zero net benefit? Pharmacoeconomics. Forthcoming $201 \mathrm{I}$.

6 Rawlins M, Barnett D, Stevens A. Pharmacoeconomics: NICE's approach to decision-making. Br J Clin Pharmacol 2010; 70:346-49. doi: I0.1 III/j.1365-2I25.2009.03589.x

7 National Institute for Health and Clinical Excellence. Cetuximab for the treatment of recurrent and/or metastatic squamous cell cancer of the head and neck. NICE technology appraisal guidance 172. London: NICE; 2009. [cited 20II June I2]. Available from: http://www.nice. org.uk/nicemedia/pdf/TAI72Guidance.pdf
A further argument is that the cost per QALY threshold is too low at $£ 20,000-£ 30,000$ per QALY relative to another standard treatment and that a higher value is warranted. This calls for a closer examination of this threshold. It is used for decision-making purposes to represent the marginal value of a QALY, that is, if all NHS interventions and services were ranked from the most to the least efficient, the marginal QALY is the value of the one ranked lowest. It is not something which can be increased or decreased at the discretion of NICE but is generally defined by reasonable assessments of value over several sets of appraisals over time. There are uncertainties in specifying the threshold value, hence the adopted range of $£ 20,000$ to $£ 30,000$ per QALY, but even the lower end may be too high when compared with actual NHS activities," ${ }^{11,12}$ which means we are enabling treatments to be used in the NHS that are inefficient. Thus the effect of the NICE end of life criteria and the ring-fencing afforded by the Cancer Drugs Fund in England is to further directly detract from provision of other areas of care in the NHS: can we afford this in the current cold economic climate and within the restraints created by a contracting public sector?

8 Clement FM, Harris A, Li JJ et al. Using effectiveness and costeffectiveness to make drug coverage decisions: a comparison of Britain, Australia, and Canada. JAMA 2009; 302:I437-43. doi: I0.100I/jama.2009.1409

9 Mason A, Drummond M, Ramsey S et al. Comparison of anticancer drug coverage decisions in the United States and United Kingdom: does the evidence support the rhetoric? J Clin Oncol 2010; 28:3234-38. doi: $10.1200 / J C O .2009 .26 .2758$

10 Richards M. Improving access to medicines for NHS patients. A report for the Secretary of State for Health by Professor Mike Richards CBE. 2008. [cited 20I I June 12]. Available from: http://www.dh.gov.uk/ prod_consum_dh/groups/dh_digitalassets/@dh/@en/documents/ digitalasset/dh_089952.pdf

II Martin S, Rice N, Smith PC. Does health care spending improve health outcomes? Evidence from English programme budgeting data. J Health Econ 2008; 27:826-42. doi: 10.1016/j.jhealeco.2007.12.002

12 Raftery J. Should NICE's threshold range for cost per QALY be raised? No. BMJ 2009; 338:b I85. doi: I0.1136/bmj.bI85 